\title{
Gingival Crevicular Fluid in Periodontitis
}

Dr. Surendra Man Shrestha

Keywords: Diagnosis; gingival crevicular fluid; periodontitis.

Gingival crevicular fluid (GCF) is an inflammatory exudate that is derived from the periodontal tissue and can be collected at the orifices or from within the gingival crevice. ${ }^{1}$ GCF is an exudate released as a result of inflammation in the tissue adjacent to the crevicular epithelium. It has been shown that clinically healthy gingiva always shows histological evidence of inflammation by accumulation of polymorphonuclear leukocytes (PMNLs). This is because some amount of plaque is always present in sulcular region which irritates the sulcular epithelium and the GCF accumulates in the gingival sulcus.

The fluid portion of the exudate is derived from microleakage of the subgingival microvasculature. The constituent of GCF are derived from a number of sources including serum, connective tissue and both host cells and microorganisms in the gingival crevice.

The periodontal diagnostic importance of the GCF was recognized more than 60 years ago, but serious investigation of dynamics of the GCF production began in in the late 1950s by Brill and co-workers. ${ }^{2}$ The fact that GCF can be harvested from the gingival sulcus offers great potential as a source of factors associated with disease and destruction. The analysis of GCF for disease marker has numerous advantages, because unlike serum and saliva, it is site-specific, conveniently sampled and non-invasive. Tissue destruction as a consequence of host-bacterial interaction is a well described process in the pathogenesis of periodontal diseases. During the destruction of host cells (mainly PMNLs) release their granular enzymes that are capable of attacking all extracellular matrix components. Thus, extracellular presence of enzymes seems to play an important role in the connective tissue damage.

Analysis of these enzymes in GCF may lead to insight into the pathogenesis of periodontal disease and may provide rational basis for the development of novel diagnostic tests. The enzymes are acid phosphatase, alkaline phosphatase, beta-glucuronidase, lysozyme, hyaluronidase, cathepsin, collagenase, lactic dehydrogenase, etc.
Host responses to periodontal infections include the production of several families of enzymes that are released by microorganism, epithelial or inflammatory cells. Enzymes synthesised and secreted in the tissue are carried by GCF to the crevice where they are augmented by release of enzymes from bacteria and host cells present in pocket.

The diagnostic potential of GCF has been studied for several years. ${ }^{3}$ However recently there has been renewed interest in analysing the constituents of GCF using non-invasive methods, by which the host response as well as disease activity in periodontium can be elicited. The constituents of GCF include cellular elements, electrolytes, organic compounds, bacterial products, metabolic products and enzymes. Studies on enzymatic content of GCF is of great diagnostic value since enzymes play an important role in pathogenesis of periodontal disease.

The assessment of periodontal disease and the effectiveness of periodontal therapy have traditionally been made using clinical and radiographic parameters. Determination of variables such as probing depth, sulcular bleeding following the probing and height of alveolar crest are the traditional basis of periodontal evaluation and periodontal treatment plan. Nevertheless, recent advances in understanding of the natural history of periodontal disease have raised question about the significance of these diagnostic criteria. ${ }^{4}$ As chronic disorder, patient with periodontitis will experience both active and inactive phases. Enzymes in GCF can serve as a marker for primary granules released from PMNLs. The presence of these enzymes in GCF was able to identify and predict probing attachment loss in periodontitis. ${ }^{5,6}$

\section{Correspondence: \\ Dr. Surendra Man Shrestha \\ Journal of Nepalese Society of Periodontology and Oral \\ Implantology, Kathmandu, Nepal. \\ email: surendra12000@gmail.com}

\section{Citation}

Shrestha SM. Gingival crevicular fluid in periodontitis. J Nepal Soc Perio Oral Implantol. 2018;2(2):33

\section{REFERENCES}

1. Lamster IB. The host response in gingival crevicular fluid: potential applications in periodontitis clinical trials. J Periodontol. 1992 Dec;63(12 Suppl):1117-23.

2. Cimasoni G. Crevicular fluid updated. Monogr Oral Sci. 1983;12:III-VII,1-152.

3. Ghallab NA. Diagnostic potential and future directions of biomarkers in gingival crevicular fluid and saliva of periodontal diseases: Review of the current evidence. Arch Oral Biol. 2018;87:115-24.

4. Bostanci N, Belibasakis GN. Gingival crevicular fluid and its immune mediatiors in the proteomic era. Periodontol 2000. 2018;76:68-84.

5. Lamster IB, Oshrain RL, Harper DS, Celenti RS, Hovliaras CA, Gorden JM. Enzyme activity in crevicular fluid for detection and prediction of clinical attachment loss in patients with chronic periodontitis. J Periodontol. 1988 Aug;59(8):516-23.

6. Lamster IB, Oshrain HL, Celenti RS, Fine JB, Grbic JT. Indicators of the acute inflammatory and humoral responses in gingival crevicular fluid: relationship to active periodontal disease. J Periodontal Res. 1991 May:26(3 Pt 2):261-3. 